\title{
APROVECHAMIENTO DE ROCA FOSFÓRICA, POR VÍA TÉRMICA, PARA LA OBTENCIÓN DE TERMOFOSFATOS
}

EXPLOITATION OF PHOSPHORIC ROCK BY THERMAL MEANS TO OBTAIN THERMOPHOSPHATES

Nelson Mauricio Espinel Pérez*

https://orcid.org/0000-0002-1462-1989

CENTRO MINERO - REGIONAL BOYACÁ, SENA, COLOMBIA

In Memoriam: Efrén de Jesús Muñoz Prieto**

Recibido: 24 de octubre de 2019

Aceptado: 15 de diciembre de 2019

DOI: https://doi.org/10.29097/2011-639X.266

\section{Resumen}

Este trabajo pretende documentar el aprovechamiento de roca fosfórica para la obtención de termofosfatos a partir de fuentes de fosforita provenientes de los municipios de Iza y Sogamoso, Boyacá. El informe de la IFA indica que la demanda global de fertilizantes en relación con el P2O5 fue de 45,4 Mt en 2019, y las perspectivas de los fosfatos para la obtención de productos podrían llegar a 112 Mt para el año 2022. Para lograr este objetivo, se reporta el método de calcinación en horno eléctrico como una de las técnicas más comunes para la concentración de P2O5. En tal sentido se aplicó un diseño de experimentos factorial 23, con dos niveles (bajo y alto) y tres factores: temperatura $\left(900^{\circ} \mathrm{C}\right.$ y $\left.1200^{\circ} \mathrm{C}\right)$, tiempo de calcinación (3 y 5 horas) y fuente del mineral (RFIZA y RFPC); el tamaño de partícula $(\leq 0,149 \mathrm{~mm})$ se mantuvo constante. Los resultados obtenidos muestran un incremento de la concentración de fósforo en las muestras procesadas, especialmente las que fueron sometidas a mayores tiempos de calcinación y mayores temperaturas.

Palabras clave: apatitas, hornos eléctricos, roca fosfórica, termofosfatos.

\section{Abstract}

This work intends to document the use of phosphoric rock to obtain thermophosphates, from phosphorus sources, from the municipalities of Iza and Sogamoso, Boyacá. The IFA report indicates that the global demand for fertilizers in relation to P2O5 was 45,4 Mt in 2019, and the prospects for phosphates to obtain products could reach $112 \mathrm{Mt}$ by 2022. To achieve this purpose, the method of calcination in an electric oven is reported as one of the most common techniques for the concentration of P2O5. In this sense, a design of factorial experiments 23 was applied, with two levels (low and high) and three factors: temperature $\left(900^{\circ} \mathrm{C}\right.$ and $1200^{\circ} \mathrm{C}$ ), calcination time (3 and 5 hours) and source of the mineral (RFIZA and RFPC); the particle size $(\leq 0,149 \mathrm{~mm})$ remains constant. The results obtained show an increase in phosphorus concentration in the processed samples, especially those that were subject of higher calcination times and higher temperatures.

Keywords: apatite, electric ovens, phosphoric rock, thermophosphates.

\footnotetext{
* Máster en Química, Grupo de investigación Desarrollo y aplicaciones de nuevos materiales (DANUM), Universidad Tecnológica y Pedagógica de Colombia, Tunja - Boyacá, Colombia. Grupo GICEMIN, Centro Minero - Regional Boyacá, SENA, Colombia. « espinelson@misena.edu.co

** [Profesor Asociado Uptc], [DANUM], Coordinador Grupo de Investigación Danum, Escuela de Ciencias Químicas, Universidad Pedagógica y Tecnológica de Colombia, Tunja- Boyacá, Colombia.
} 


\section{INTRODUCCIÓN}

Los cinco tipos de recursos minerales fosfatados que se pueden encontrar alrededor del mundo son: depósitos de fosfatos marinos, fosfatos ígneos, metamórficos, biogénicos y fosfatos producto del desgaste (Straaten, 2002). Cerca del $75 \%$ de las rocas fosfóricas del mundo son de origen sedimentario, y el $75 \%$ al $80 \%$ contienen ganga de carbonato (Shariati et al., 2015). De acuerdo con Abouzeid (2008), la mineralogía de las rocas fosfatadas las podemos encontrar según la descripción de los depósitos naturales: la fluorapatita $\left[\mathrm{Ca}_{10}\left(\mathrm{PO}_{4}\right)_{6} \mathrm{~F}_{2}\right]$ se encuentra principalmente en rocas ígneas y entornos metamórficos, por ejemplo, en carbonatitas y mica-piroxenitas; la hidroxiapatita $\left[\mathrm{Ca}_{10}\left(\mathrm{PO}_{4}\right)_{6}(\mathrm{OH})_{2}\right]$ se encuentra sobre todo en ambientes ígneos y metamórficos, así como en depósitos biogénicos como, por ejemplo, en depósitos de huesos; el carbonato de hidroxiapatita $\left[\mathrm{Ca}_{10}\left(\mathrm{PO}_{4}, \mathrm{CO}_{3}\right)_{6}(\mathrm{OH})_{2}\right]$, también llamado guano, se encuentra principalmente en las islas y en las cuevas, como parte de los excrementos de las aves y murciélagos; la francolita [Ca10-x-y $\mathrm{Na}_{\mathrm{x}} \mathrm{Mg}_{\mathrm{y}}\left(\mathrm{PO}_{4}\right)_{6-}$ ${ }_{\mathrm{z}}\left(\mathrm{CO}_{3}\right)_{\mathrm{z}} \mathrm{F}_{0 .{ }_{\mathrm{z}}} \mathrm{F}_{2}$ ], complejo de carbonato apatita-sustituido, se encuentra solo en ambientes marinos, y en menor medida en los depósitos temporales, como las carbonatitas; la dahlita $\left[3 \mathrm{Ca}_{3}\left(\mathrm{PO}_{4}\right)_{2} \cdot \mathrm{CaCO}_{3}\right]$ es una estructura de fosfato que se encuentra en los sedimentos marinos; finalmente, la colofona $\left[3 \mathrm{Ca}_{3}\left(\mathrm{PO}_{4}\right)_{2} \cdot \mathrm{nCa}\right.$ $\left.\left(\mathrm{CO}_{3}, \mathrm{~F}_{2}, \mathrm{O}\right) \cdot \mathrm{xH}_{2} \mathrm{O}\right]$ es un tipo de minerales de fosfato típico de los sedimentos marinos.

El grupo más importante de los fosfatos presentes en las rocas ígneas, sedimentarias y metamórficas es el de las apatitas $\left[\mathrm{Ca}_{5}(\mathrm{~F}, \mathrm{Cl}, \mathrm{OH}) *\left(\mathrm{PO}_{4}\right)_{3}\right]$, ya que representa una mezcla entre fluorapatita $\left[\mathrm{Ca}_{5}(\mathrm{~F}) \cdot\left(\mathrm{PO}_{4}\right)_{3}\right]$, cloroapatita $\left[\mathrm{Ca}_{5}\right.$ $\left.(\mathrm{Cl}) \cdot\left(\mathrm{PO}_{4}\right)_{3}\right]$ e hidroxiapatita $\left[\mathrm{Ca} 5(\mathrm{OH})\left(\mathrm{PO}_{4}\right)_{3}\right]$ (ver tabla 1$)$.

\section{MARCO TEÓRICO}

\section{Tratamientos térmicos}

Blazy \& Bouhaouss (2005) reportan que el $10 \%$ de los fosfatos comercializables en el mundo se producen por calcinación, en una relación con la disociación térmica de carbonatos y la combustión de materias orgánicas en los minerales. Estos tratamientos térmicos podrían ser aplicados a la roca fosfórica proveniente de los municipios de Iza y Sogamoso (Boyacá), pues el objetivo del presente trabajo es la obtención de termofosfatos, y, a la luz de los estudios realizados por Unión Temporal GI. Georecursos (2005), los yacimientos de roca fosfórica de Boyacá pueden "ser utilizados como materia prima para la fabricación de fertilizantes, en especial ácido fosfórico y termofosfatos”. 


\section{Tabla 1}

Generalidades sobre las apatitas

\begin{tabular}{|c|c|}
\hline Fórmula general & $\mathrm{M}_{10}\left(\mathrm{XO}_{4}\right)_{6} \mathbf{Y}_{2 .}$ \\
\hline M (Cationes a sustituir) & $\begin{array}{l}\mathrm{Ca}^{2+}, \mathrm{Mg}^{2+}, \mathrm{Sr}^{2+}, \mathrm{Ba}^{2+}, \mathrm{Mn}^{2+}, \mathrm{Fe}^{2+}, \mathrm{Zn}^{2+}, \mathrm{Cd}^{2+}, \mathrm{Pb}^{2+}, \mathrm{H}^{+}, \mathrm{Na}^{+}, \\
\mathrm{K}^{+}, \mathrm{Al}^{3+} \text {. }\end{array}$ \\
\hline $\mathrm{XO}_{4}$ (Aniones a sustituir) & $\mathrm{PO}_{4}^{3-}, \mathrm{AsO}_{4}{ }^{3-}, \mathrm{VO}_{4}{ }^{3-}, \mathrm{SO}_{4}{ }^{3-}, \mathrm{CO}_{3}{ }^{2-}$ y $\mathrm{SiO}_{4}{ }^{3-}$ \\
\hline Y (Aniones a sustituir) & $\mathrm{OH}^{-}, \mathrm{F}^{-}, \mathrm{Cl}^{-}, \mathrm{Br}^{-}, \mathrm{O}^{2-}, \mathrm{y} \mathrm{CO}_{4}^{2-}$ \\
\hline Estabilidad térmica & FAp $>$ HAp $>$ ClAp \\
\hline $\begin{array}{l}\text { Grado de disolución en } \\
\text { tampón ácido }\end{array}$ & ClAp $>$ HAp $>$ FAp \\
\hline
\end{tabular}

Nota. La tabla resume algunas generalidades sobre las apatitas, las cuales sirven para inferir características de su composición química. Tomado de: Fahami, Bahman \& Reza (2013); Legeros, Ito, Ishikawa, Sakae \& Legeros (2009); Kijkowska, Lin \& Legeros (2002).

Uno de los ejemplos puntuales sobre la descomposición térmica de las apatitas lo podemos encontrar en un proceso llamado deshidroxilación de la hidroxiapatita, el cual consiste en la pérdida de grupos hidroxilo tal como se muestra en la siguiente reacción (Levingstone, 2008):

$$
\begin{aligned}
& \mathrm{Ca}_{10}\left(\mathrm{PO}_{4}\right)_{6}(\mathrm{OH})_{2} \rightarrow \mathrm{Ca}_{10}\left(\mathrm{PO}_{4}\right)_{6} \mathrm{OH}_{2-2 X} \mathrm{O}_{X \boxminus X}+x \mathrm{H}_{2} \mathrm{O} \\
& \text { Hidroxiapatita (HA) Oxihidroxiapatita (OHA) } \\
& \mathrm{Ca}_{10}\left(\mathrm{PO}_{4}\right)_{6} \mathrm{OH}_{2-2 X} \mathrm{O}_{X \square X}+x \mathrm{H}_{2} \mathrm{O} \rightarrow \mathrm{Ca}_{10}\left(\mathrm{PO}_{4}\right)_{6} \mathrm{O}_{X \boxminus X}+(1-x) \mathrm{H}_{2} \mathrm{O} \\
& \text { Oxihidroxiapatita }(\mathrm{OHA}) \quad \text { Oxiapatita }(\mathrm{OA})
\end{aligned}
$$

En la primera etapa (reacción 1 y 2), se da la formación de oxihidroxiapatita, que es un producto deficiente de iones hidroxilo, y de oxiapatita, las cuales se pueden transformar nuevamente en HA ante la presencia de agua (Bernal Gaona, 2013).

$$
\begin{array}{ccc}
\mathrm{Ca}_{10}\left(\mathrm{PO}_{4}\right)_{6} \boldsymbol{O}_{X \square X} & \rightarrow & \mathbf{2 C a}_{3}\left(\mathrm{PO}_{4}\right)_{2}+\mathrm{Ca}_{4} \mathrm{O}\left(\mathrm{PO}_{4}\right)_{2} \\
\text { Oxiapatita }(O A) & \text { Fosf. tricálcico (TCP) + Fosf. tetracálcico (TTCP) }
\end{array}
$$


Bernal Gaona (2013) indica que cuando se superan las temperaturas por encima de cierto punto crítico, se da una deshidroxilación completa e irreversible, y la oxiapatita se transforma en fosfato tricálcico y tetracálcico, que a su vez se pueden transformar en $\mathrm{CaO}$ y $\mathrm{P}_{2} \mathrm{O}_{5}$.

$$
\begin{array}{ccc}
\mathrm{Ca}_{3}\left(\mathrm{PO}_{4}\right)_{2} & \rightarrow & \mathbf{3 C a O}+\mathbf{P}_{\mathbf{2}} \mathbf{0}_{\mathbf{5}} \uparrow \\
\text { Fosfato tricálcico (TCP) } & \text { Óxido de calcio }+ \text { Pentóxido de fósforo } \\
\mathrm{Ca}_{4} \mathbf{O}\left(\mathrm{PO}_{4}\right)_{2} & \rightarrow & \mathbf{4 C a O}+\mathbf{P}_{\mathbf{2}} \mathbf{0}_{\mathbf{5}} \uparrow \\
\text { Fosfato tetracálcico (TTCP) } & \text { Óxido de calcio }+ \text { Pentóxido de fósforo }
\end{array}
$$

Esta descomposición térmica por calcinación facilita la concentración de fósforo, el cual se reporta como $\mathrm{P}_{2} \mathrm{O}_{5}$ y es el componente fundamental para la obtención de ácido fosfórico o de fertilizantes. Abouzeid (2008) reporta las características de un proceso de calcinación y descomposición de las apatitas, el cual aparece en la tabla 2.

\section{Tabla 2}

Tratamientos térmicos aplicados a las apatitas

\begin{tabular}{lll}
\hline i. Remoción de agua & $\mathbf{1 2 0 - 1 5 0}{ }^{\circ} \mathrm{C}$ & Secado \\
\hline ii. Remoción de materia orgánica & $650-750^{\circ} \mathrm{C}$ & Calcinación \\
\hline iii. Disociación de carbonatos & $850-1000^{\circ} \mathrm{C}$ & Calcinación \\
\hline iv. Remoción de flúor & & \\
\hline
\end{tabular}

Nota. Proceso de calcinación y descomposición de las apatitas según Abouzeid (2008).

Un análisis termogravimétrico (DSC-TG) de roca fosfórica proveniente del norte de Arabia Saudita reporta que la deshidratación aparece a los $160^{\circ} \mathrm{C}$, acompañado por una pérdida de peso, de acuerdo con la siguiente reacción referida por Aissa et al. (2014):

$$
2 \mathrm{HPO}_{4}^{2-} \rightarrow \mathrm{P}_{2} \mathrm{O}_{7}^{4-}+\mathrm{H}_{2} \mathrm{O}
$$


Una segunda pérdida de peso aparecería a $\operatorname{los} 550^{\circ} \mathrm{C}$, tal como lo indica Elgharbi et al. (2015), debido a la descomposición de carbonatos (calcita $\left[\mathrm{CaCO}_{3}\right]$ y dolomita $\left.\left[\mathrm{CaMg}\left(\mathrm{CO}_{3}\right)_{2}\right]\right)$, con liberación de $\mathrm{CO}_{2}$. Este gas también se libera debido a la presencia de materia orgánica (figura 1) durante el proceso de calcinación. Tonsuaadu (2012) reporta los eventos térmicos de las apatitas FAp, ClAp, HAp, $\mathrm{CO}_{3} \mathrm{Ap}$ (carbonatoapatita) y BioAp (bio-apatita).

\section{Figura 1.}

Eventos térmicos para las apatitas FAp, ClAp, HAp, CO3Ap y BioAp.

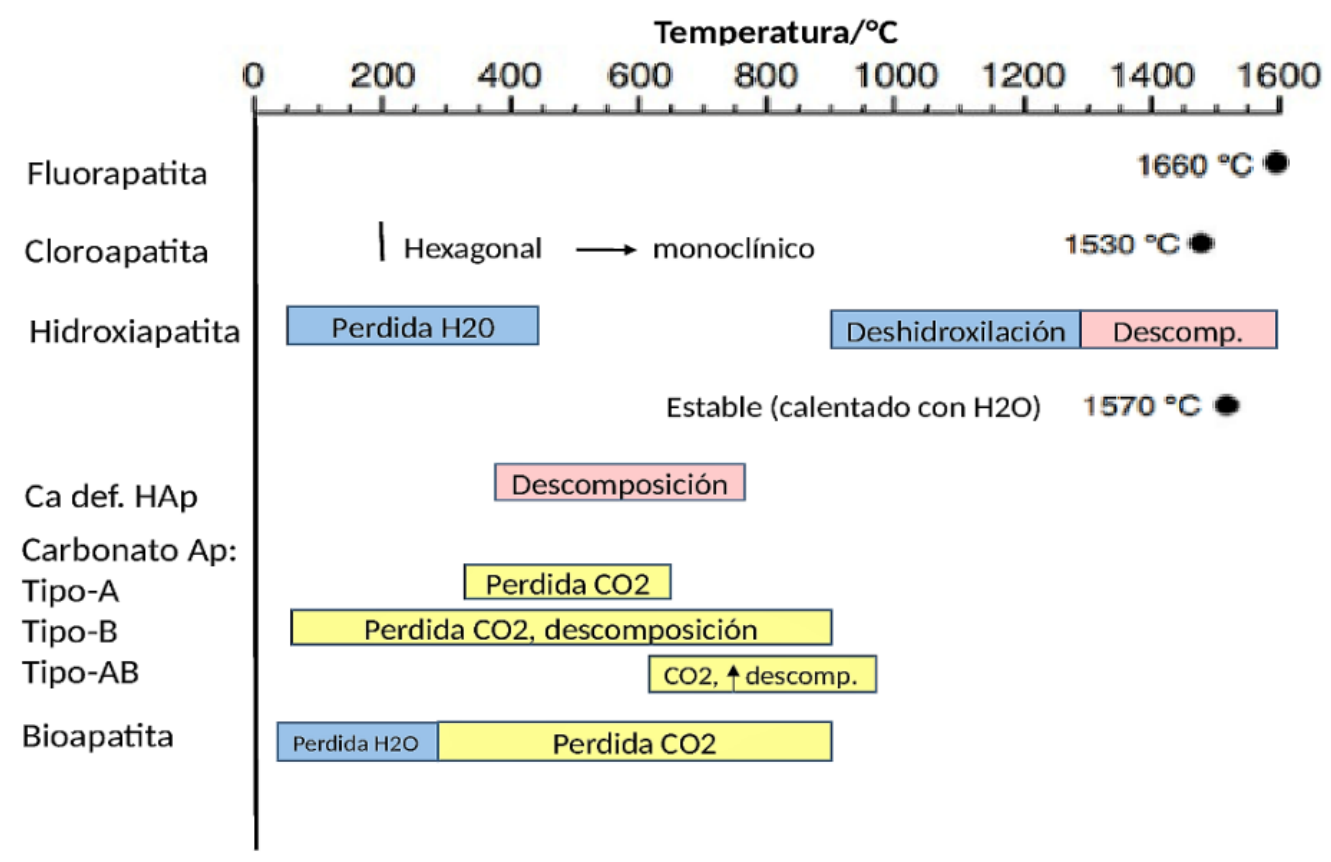

Nota. En la figura se pueden observar cambios de fase (ClAp), pérdida de grupos estructurales, descomposición y pérdidas de $\mathrm{CO}_{2}$ y $\mathrm{H}_{2} \mathrm{O}$.

Si la roca fosfórica se calcina durante un tiempo determinado a cierta temperatura (3h- $1000^{\circ} \mathrm{C}$ ), como lo indica Calle Castañeda (2016), esta se puede descomponer hasta el 90 \% de la ganga calcárea y dolomítica, dependiendo del tamaño y la naturaleza del material. Otros parámetros relevantes de la calcinación para tener en cuenta son las propiedades fisicoquímicas de los fosfatos (El Ouardi, 2013). 
Espinel Pérez

Tabla 3

Procesos de calcinación para incrementar el porcentaje de $\mathrm{P}_{2} \mathrm{O}_{5}$

\begin{tabular}{|c|c|c|c|c|c|c|}
\hline \multirow[t]{2}{*}{ Fuente } & \multirow[t]{2}{*}{ Temp. $\left({ }^{\circ} \mathrm{C}\right)$} & \multirow{2}{*}{$\begin{array}{l}\text { Medio de } \\
\text { calcinación }\end{array}$} & \multirow[t]{2}{*}{ Tiempo (h) } & \multicolumn{2}{|c|}{$\% \mathrm{P}_{2} \mathrm{O}_{5}$} & \multirow{2}{*}{$\begin{array}{c}\% \text { de } \\
\text { incremento }\end{array}$} \\
\hline & & & & Inicial & Final & \\
\hline Fernández \& & 900 & Mufla & 3 & 22,33 & 25,18 & 12,76 \\
\hline Noguera & & & & 28,83 & 30,00 & 4,06 \\
\hline (2003), & & & & & & \\
\hline Venezuela & & & 5 & 22,33 & 26,05 & 16,70 \\
\hline & & & & 28,83 & 30,85 & 7,00 \\
\hline & & & 7 & 22,33 & 26,08 & 16,80 \\
\hline & & & & 28,83 & 31,22 & 8,3 \\
\hline Bojinova & 200 & Horno & 1 & 29,40 & 29,74 & 1,2 \\
\hline (2003), & 800 & eléctrico & & 29,40 & 31,50 & 7,10 \\
\hline Túnez & 1200 & & & 29,40 & 32,38 & 10,14 \\
\hline El Ouardi & 900 & Horno & 0,66 & 30,39 & 34,4 & 13,2 \\
\hline (2013), & & eléctrico & & & & \\
\hline Marruecos & & & & & & \\
\hline El-Midany et & 1100 & Horno de & $0,17-1,17$ & 24,00 & 34,00 & 41,70 \\
\hline al. (2013), & & cama & & & & \\
\hline Arabia & & estática & & & & \\
\hline
\end{tabular}


Aprovechamiento de roca fosfórica por vía térmica

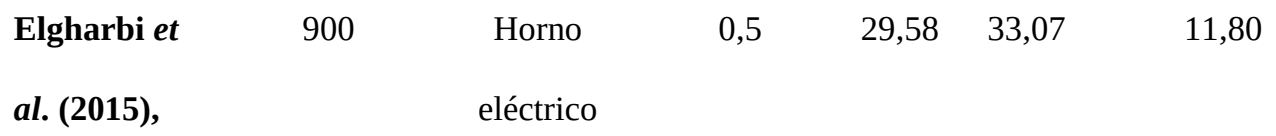

Túnez

Nota. La tabla muestra algunos procesos de calcinación alrededor del mundo, encaminados a incrementar el porcentaje de $\mathrm{P}_{2} \mathrm{O}_{5}$ respecto de su mineral original. Tomado de: Fernandez \& Noguera (2003); Bojinova (2003); El Ouardi (2013); ElMidany, El-Aleem \& Al-Fariss (2013); Elgharbi, Horchani-Naifer \& Férid (2015).

\section{Metodología}

\section{Experimentos de calcinación}

Se tomaron muestras de roca fosfórica en dos minas con diferente tenor: la primera fue tomada de la mina Montesión, perteneciente a la vereda Pilar y Ceibita (RFPC) del municipio de Sogamoso (Boyacá); la segunda muestra (RFIZA) fue tomada de la mina en la vereda San Miguel del Municipio de Iza (Boyacá). Las mismas se sometieron a procesos de beneficio de minerales, los cuales incluyeron secado, trituración, molienda y tamizado. El tamaño de partícula seleccionado para los experimentos fue de 0,074 $\mathrm{mm}$.

\section{Tabla 4}

Procesos de calcinación para incrementar el porcentaje de $\mathrm{P}_{2} \mathrm{O}_{5}$

\begin{tabular}{lccc}
\hline Nivel & Temperatura $\left({ }^{\circ} \mathbf{C}\right)$ & Tiempo (h) & Fuente \\
\hline Bajo & 900 & 3 & RFIZA \\
Alto & 1200 & 5 & RFPC \\
\hline
\end{tabular}

Nota. Para poder observar los efectos significativos de las variables experimentales se realizó un diseño factorial $2^{3}$, utilizando el programa Minitab 17; tanto los niveles como los factores se describen en la tabla.

Para cada experimento se pesaron 5,0 g de las muestras representativas de RFPC y RFIZA; luego se colocaron cada una en un crisol de porcelana y se sometieron a tiempos de calcinación de 3 y $5 \mathrm{~h}$ y a temperaturas de $900^{\circ} \mathrm{C}$ y $1200^{\circ} \mathrm{C}$, respectivamente, en una mufla marca Carbolite CWF 12/5. La rampa de calentamiento se realizó incrementando $26,7^{\circ} \mathrm{C} / \mathrm{min}$ hasta llegar a los $900^{\circ} \mathrm{C}$, en un tiempo de 34 minutos aproximadamente, y 
a los $1200^{\circ} \mathrm{C}$, en un tiempo de 45 minutos aproximadamente. Posteriormente, los crisoles de porcelana se sacaron de la mufla y fueron llevados a un desecador hasta alcanzar temperatura ambiente.

\section{Equipos e instrumentos}

Los métodos analíticos instrumentales utilizados fueron: espectrofotometría UVVis, difracción de rayos X (DRX), microscopía electrónica de barrido (SEM) y termogravimetría (TGA).

Para la determinación de fósforo total presente en las muestras calcinadas se usó el método espectrofotométrico del fosfomolibdovanadato a 400nm (Norma Técnica Colombiana [NTC 234], 2001), mediante un espectrofotómetro UV-VIS Shimadzu 1601.

Los análisis DRX fueron llevados a cabo usando un equipo GNR XRD 600, con

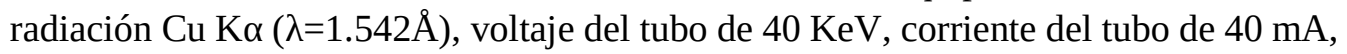

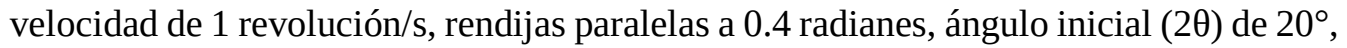

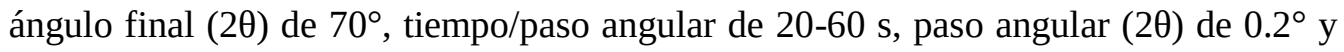
tiempo de integración de 35 minutos.

El software utilizado para identificar las fases cristalinas fue Xpert Highscore versión 3.0 de PANalytical, con el cual se realizó un refinamiento rietveld para todas las muestras.

Con respecto a la materia prima sin calcinar (RFPC y RFIZA), se realizaron análisis utilizando la técnica de microscopia electrónica de barrido (SEM), mediante un equipo marca ZEIIS Serie EVO MA10.

En cuanto a los análisis termogravimétricos (TGA), se utilizó un equipo marca LECO 701 y bajo la norma ASTM D7582, con el cual se determinó humedad, materia volátil, cenizas y carbono fijo.

\section{Resultados}

Uno de los resultados físicos de la calcinación de cada muestra, a $900^{\circ} \mathrm{C}-1200^{\circ} \mathrm{C}$, y utilizando diferentes tiempos de residencia (3-5 h), fue el cambio físico de color, tal como se observa en las siguientes figuras 2, 3 y 4 : 
Figura 2.

Muestras crudas RFIZA, RFPC y termo- Termofosfatos E3 (A), E4 (A), E5 (B) y E6 (B). fosfatos E1 (A) y E2 (A).
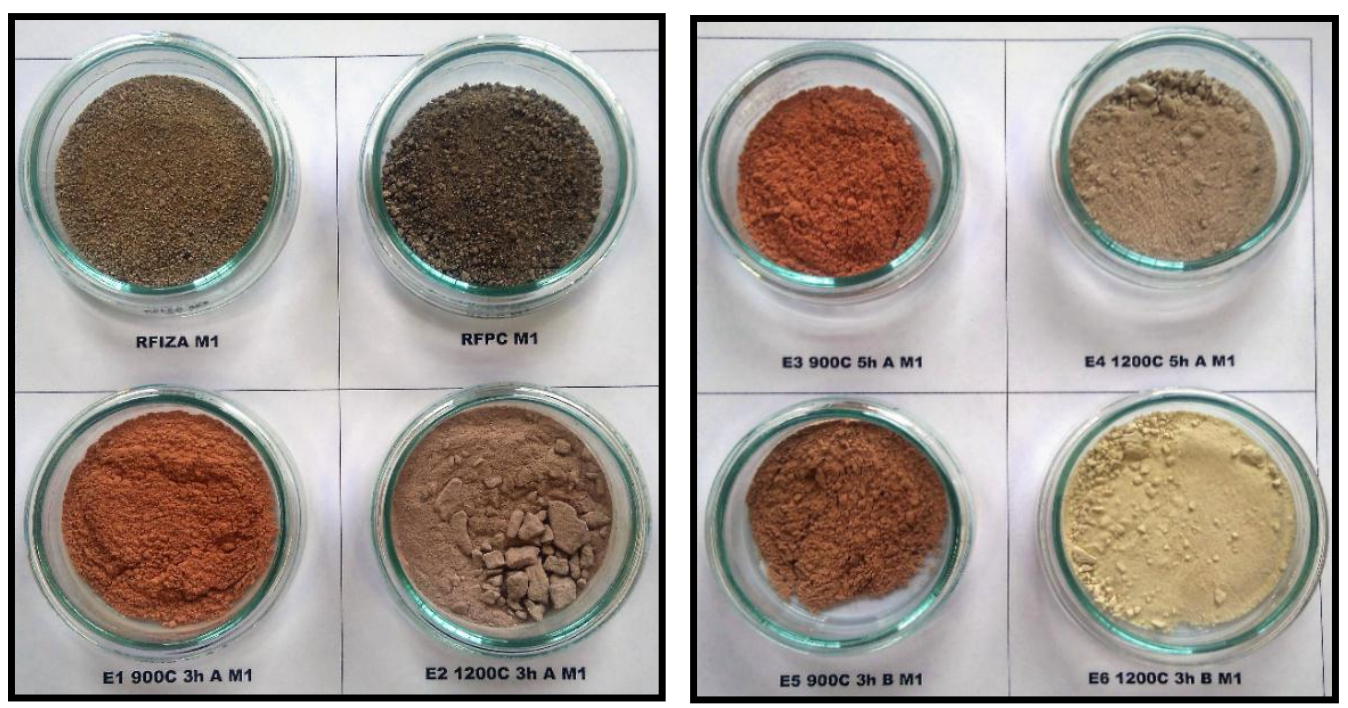

Figura 4.

Termofosfatos E7 (B) y E8 (B).

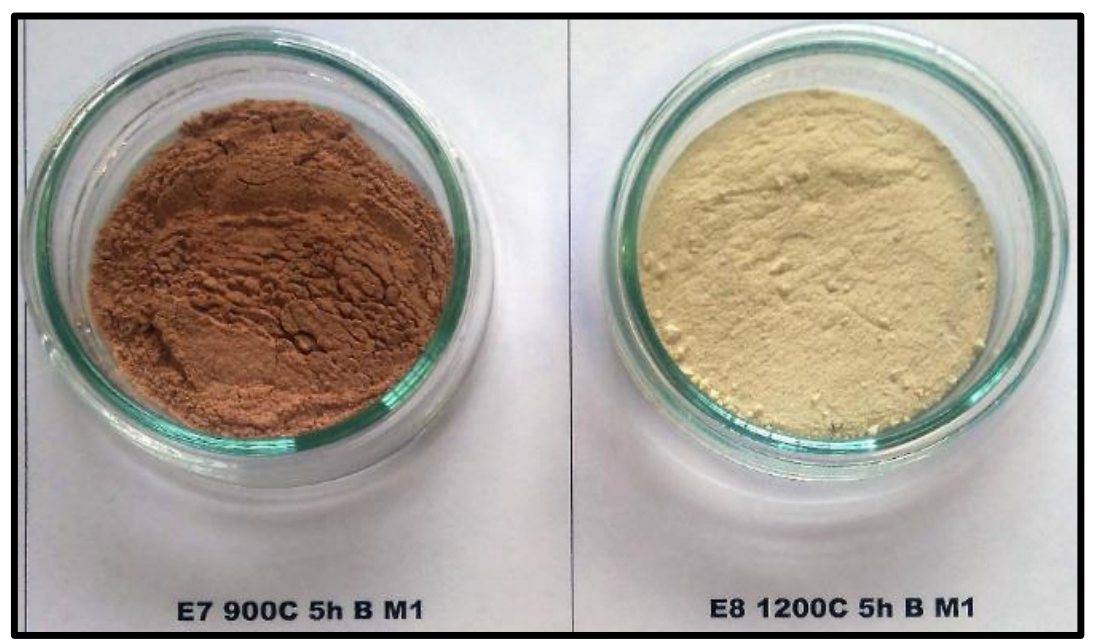

Para la determinación de fosfatos por el método espectrofotométrico se desarrolló una curva de calibración, tal como se indica en la figura 5 a partir de los datos suministrados en la tabla 5: 


\section{Espinel Pérez}

\section{Tabla 5}

Curva de calibración de $\mathrm{P}_{2} \mathrm{O}_{5}$

\begin{tabular}{cc}
\hline $\mathbf{m g} \mathbf{P}_{2} \mathbf{O}_{5}$ & Absorbancia \\
\hline 2 & 0,000 \\
2,5 & 0,176 \\
3 & 0,386 \\
3,5 & 0,576 \\
4 & 0,753 \\
4,5 & 0,896 \\
5 & 1,049 \\
\hline
\end{tabular}

Figura 5.

Curva de calibración de fosfatos

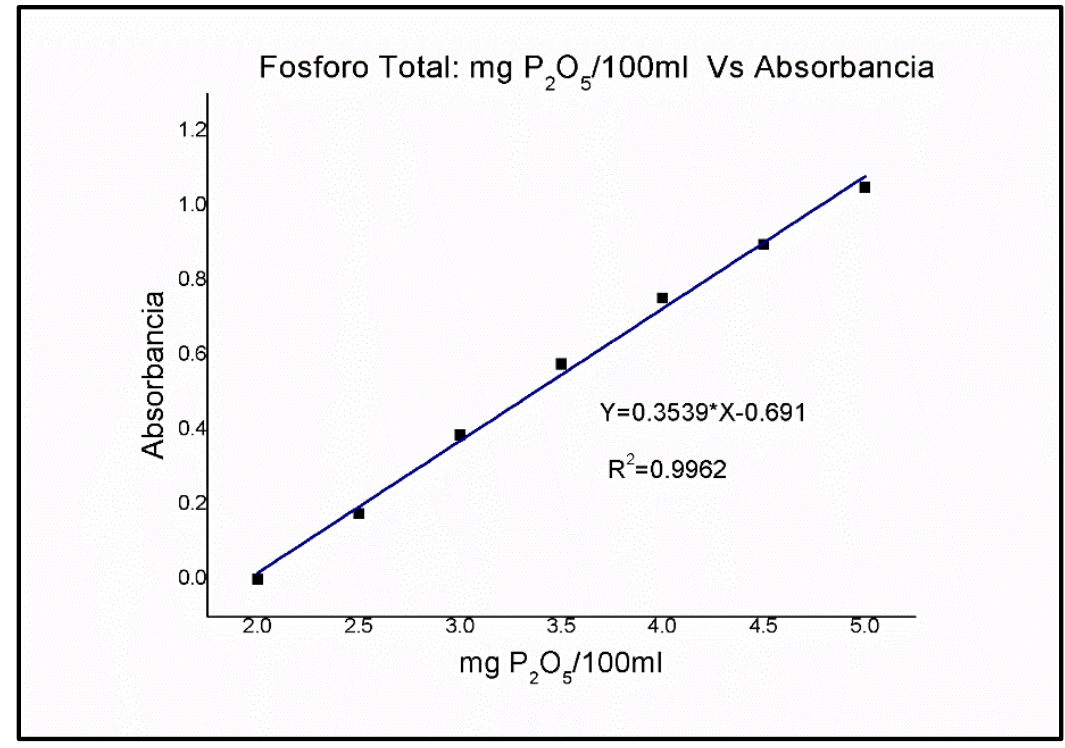

Nota. NTC 234, 2001; espectrofotómetro Shimadzu UV-VIS 1601. 
Los resultados correspondientes al incremento de porcentaje de $\mathrm{P}_{2} \mathrm{O}_{5}$ después de la calcinación se muestran en la tabla 6:

\section{Tabla 6}

Incremento de porcentaje de $\mathrm{P}_{2} \mathrm{O}_{5}$ después de la calcinación.

\begin{tabular}{lcc}
\hline \multicolumn{1}{c}{ Muestra } & \% de $\mathbf{P}_{2} \mathbf{O}_{\mathbf{5}}$ & $\begin{array}{c}\text { Incremento } \\
\text { porcentual de } \mathbf{P}_{2} \mathbf{O}_{5}\end{array}$ \\
\hline RFIZA Cruda & 23,14 & \\
\hline E1 900C 3h A M1 & 24,59 & 6,27 \\
\hline E2 1200C 3h A M1 & 24,37 & 5,32 \\
\hline E3 900C 5h A M1 & 24,61 & 6,33 \\
\hline E4 1200C 5h A M1 & 25,25 & 9,10 \\
\hline RFPC Cruda & 29,94 & \\
\hline E5 900C 3h B M1 & 32,46 & 8,42 \\
\hline E6 1200C 3h B M1 & 32,22 & 7,60 \\
\hline E7 900C 5h B M1 & 32,06 & 7,10 \\
\hline E8 1200C 5h B M1 & 32,81 & 9,60 \\
\hline
\end{tabular}

Al analizar las concentraciones en la tabla 6, se observó que antes de la calcinación las muestras crudas de RFIZA y RFPC presentaron valores de 23,14 \% de $\mathrm{P}_{2} \mathrm{O}_{5}$ y 29,94 \% de $\mathrm{P}_{2} \mathrm{O}_{5}$, respectivamente. Los incrementos porcentuales después de la calcinación para las muestras E4 y E8, bajo condiciones de $1200^{\circ} \mathrm{C}$ y 5 horas como tiempo de residencia, reportaron un incremento de 9,08\% de $\mathrm{P}_{2} \mathrm{O}_{5}$ y 9,6 \% de $\mathrm{P}_{2} \mathrm{O}_{5}$, respectivamente. Esto nos indica que, bajo estas condiciones extremas, se obtienen incrementos significativos de $\mathrm{P}_{2} \mathrm{O}_{5}$, cercanos al $10 \%$ en los termofosfatos, favoreciendo la concentración de los fertilizantes y mejorando la disponibilidad en las plantas. Las muestras E1, E2 y E3 presentaron un incremento medio de $5,97 \%$ de $\mathrm{P}_{2} \mathrm{O}_{5}$, mientras que las muestras E5, E6 y E7 presentaron un incremento medio de 7,70 \% de $\mathrm{P}_{2} \mathrm{O}_{5}$.

$\mathrm{Al}$ comparar las concentraciones $\left(\mathrm{O}_{2} \mathrm{O}_{5}\right)$ de los termofosfatos obtenidos en horno eléctrico (TFHE-IZA y TFHE-PC), con los que aparecen en la tabla 3, observamos que, en estos últimos, los incrementos superiores al $10\left(\% \mathrm{P}_{2} \mathrm{O}_{5}\right)$, son debidos a mayores tiempos de calcinación, a la fuente natural de la roca fosfórica requerida y a las 


\section{Espinel Pérez}

características del tipo de horno utilizado. Los termofosfatos TFHE-IZA y TFHE-PC, alcanzan incrementos mayores o iguales al $9 \%$, debido a la naturaleza y a las propiedades fisicoquímicas de los fosfatos, según indica El Ouardi (2013). En cuanto a los análisis de DRX, las figuras 6 y 7 reportan las fases cristalinas de los termofosfatos TFHE-IZA y TFHE-PC, después del proceso de calcinación:

\section{Figura 6.}

DRX: Difractogramas termofosfatos horno eléctrico (TFHE-IZA) a $900^{\circ} \mathrm{C}$ y $1200^{\circ} \mathrm{C}$.

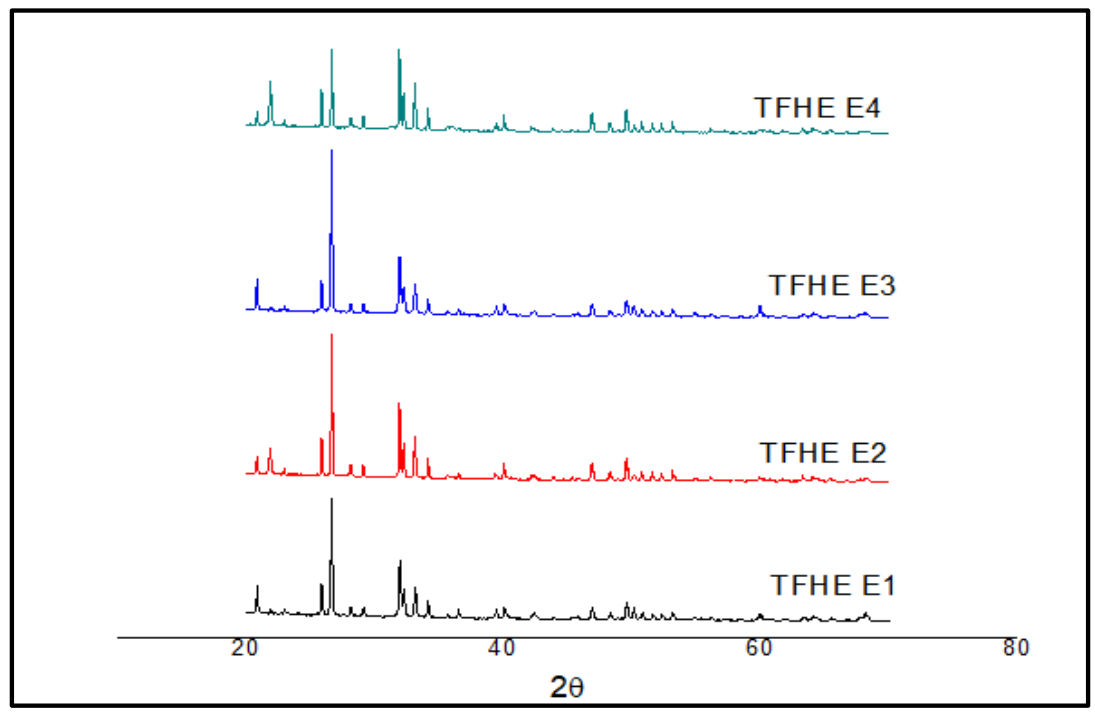

Figura 7.

DRX: Difractogramas termofosfatos horno eléctrico (TFHE-PC) a $900^{\circ} \mathrm{C}$ y $1200^{\circ} \mathrm{C}$.

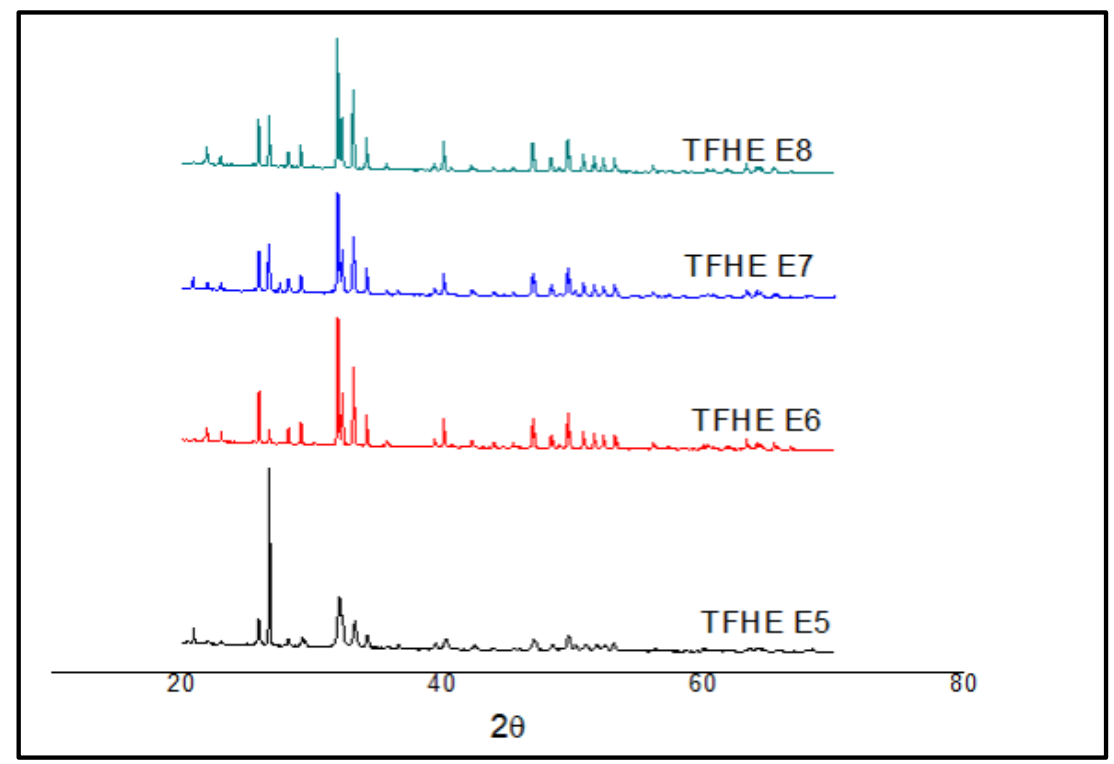


Las figuras 6 y 7, reportan los difractogramas de los termofosfatos obtenidos mediante horno eléctrico (TFHE-IZA y TFHE-PC); las fases cristalinas se identificaron mediante el uso del software Xpert Highscore versión 3.0 de PANalytical, el cual permitió la realización de un refinamiento rietveld para todas las muestras, tal como se muestra en las tablas 7 y 8.

\section{Tabla 7}

Fases Cristalinas de Termofosfatos Obtenidos en Horno Eléctrico (RFIZA).

\begin{tabular}{|c|c|c|}
\hline Muestra & $\begin{array}{c}\text { Condiciones } \\
\text { Experimentales }\end{array}$ & Fases Cristalinas \\
\hline TFHE E1 & $900^{\circ} \mathrm{C}-3 \mathrm{~h}$ & $\begin{array}{l}\text { E1 } 900 \mathrm{C} 3 \mathrm{~h} \mathrm{~A} \mathrm{M} 1 \\
\text { Apatite-(CaF) } 19.7 \% \\
\text { Apatite-(CaOH) } 80.3 \%\end{array}$ \\
\hline TFHE E2 & $1200^{\circ} \mathrm{C}-3 \mathrm{~h}$ & $\begin{array}{l}\text { E2 } 1200 \mathrm{C} 3 \mathrm{~h} \mathrm{M} 1 \\
\text { Apatite-(CaF) } 68.3 \% \\
\text { Quartz low } 2.6 \% \\
\text { Calcite } 29.0 \%\end{array}$ \\
\hline TFHE E3 & $900^{\circ} \mathrm{C}-5 \mathrm{~h}$ & $\begin{array}{l}\text { E3 900C 5h A M1 } \\
\text { Apatite-(CaF) } 44.2 \% \\
\text { Forsterite } 13.7 \% \\
\text { Coesite } 42.2 \%\end{array}$ \\
\hline TFHE E4 & $1200^{\circ} \mathrm{C}-5 \mathrm{~h}$ & $\begin{array}{l}\text { E4 } 1200 \mathrm{C} \text { 5h A M1 } \\
\text { Apatite-(CaF) } 38.5 \% \\
\text { Calcite } 16.4 \% \\
\text { Cristobalite }(\mathrm{Ca}, \mathrm{Al} \text {-bearing) } 3.0 \% \\
\text { Ellestadite-(Cl) } 42.0 \%\end{array}$ \\
\hline
\end{tabular}

En la muestra TFHE E1, se observa la presencia de Hidroxiapatita, la cual desaparece en las muestras TFHE E2, TFHE E3 y TFHE E4, debido a la eliminación de agua por el incremento de temperatura y por el tiempo de exposición. En el caso del óxido de silicio, se puede observar el cambio del sistema cristalino hexagonal (cuarzo bajo) a monoclínico (coesita), para las muestras TFHE E2 y TFHE E3. Al comparar los resultados del refinamiento rietveld (TFHE-IZA y TFHE-PC) tablas 7 y 8, podemos observar que la fluorapatita de las muestras TFHE E2, TFHE E6 y TFHE E8, presentan un mayor grado de conversión, debido a condiciones experimentales de mayor exposición y temperatura. La hidroxiapatita presente en la muestra TFHE E5, desaparece debido a estas mismas condiciones mencionadas.

En cuanto al análisis SEM para la RFIZA cruda, esta presentó morfologías y tamaños de grano de tipo globular, altamente aglomerados; los cristales están bien definidos, tal como se muestra en la figura 8 , con tamaño variable que va desde los 1043,0 $\mu \mathrm{m}$ hasta $\operatorname{los} 878,9 \mathrm{~nm}$. 


\section{Espinel Pérez}

Tabla 8

Fases Cristalinas de Termofosfatos Obtenidos en Horno Eléctrico (RFPC).

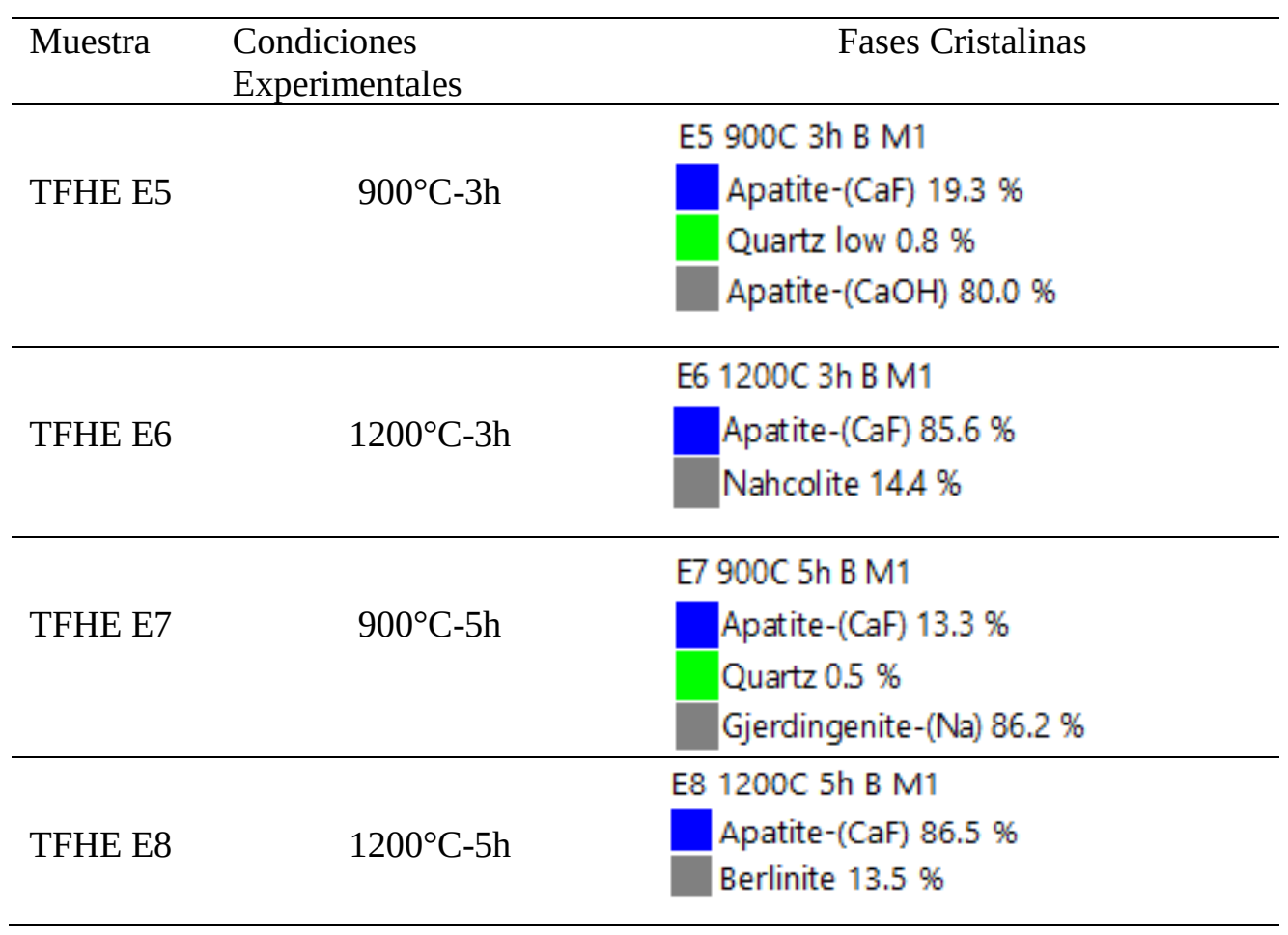

Figura 8.

SEM: Microscopía electrónica de barrido para RFIZA.

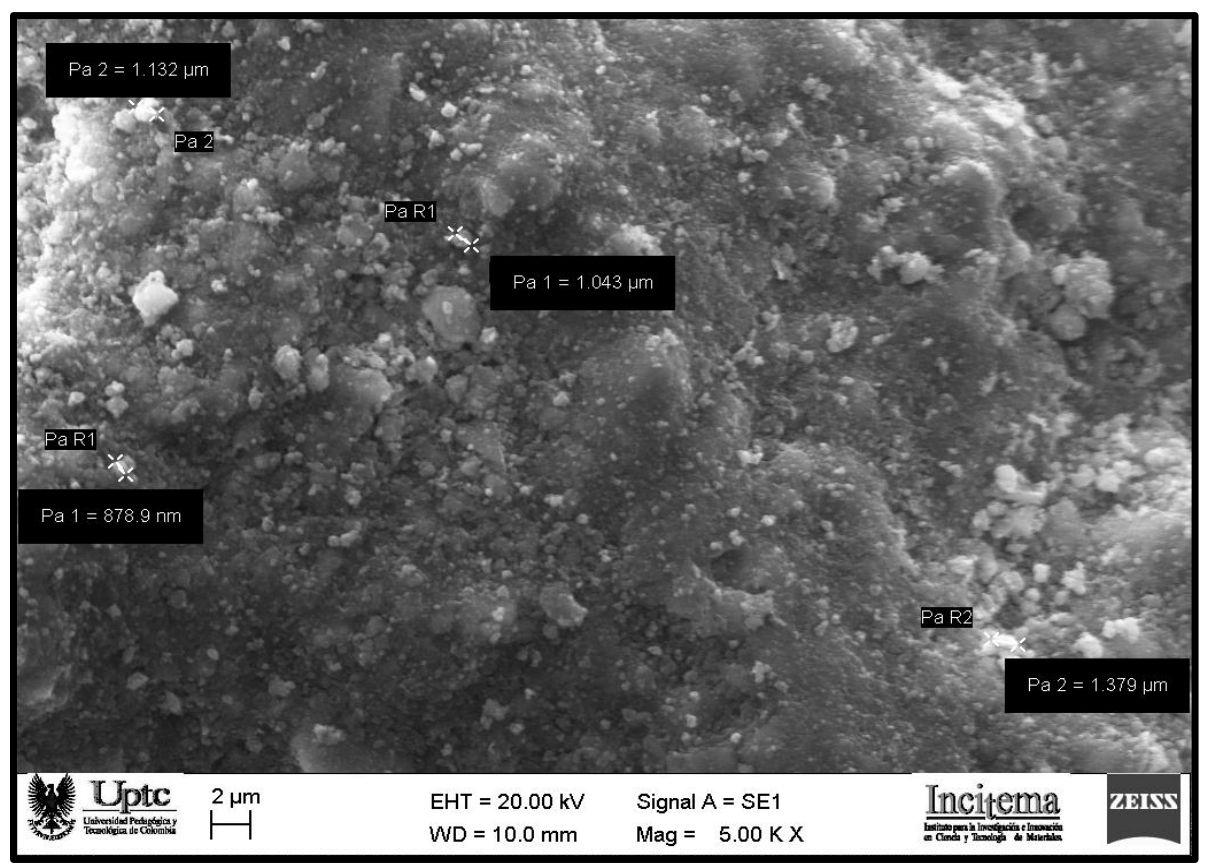


La técnica SEM acoplada a EDX, que se muestra en la figura 9 para la RFPC cruda, presentó morfologías y tamaños de grano de tipo globular, altamente aglomerados; los cristales están bien definidos, con tamaño variable que van desde 1056,0 hasta 1816,0 $\mu \mathrm{m}$.

\section{Figura 9.}

SEM: Microscopía electrónica de barrido para RFPC.

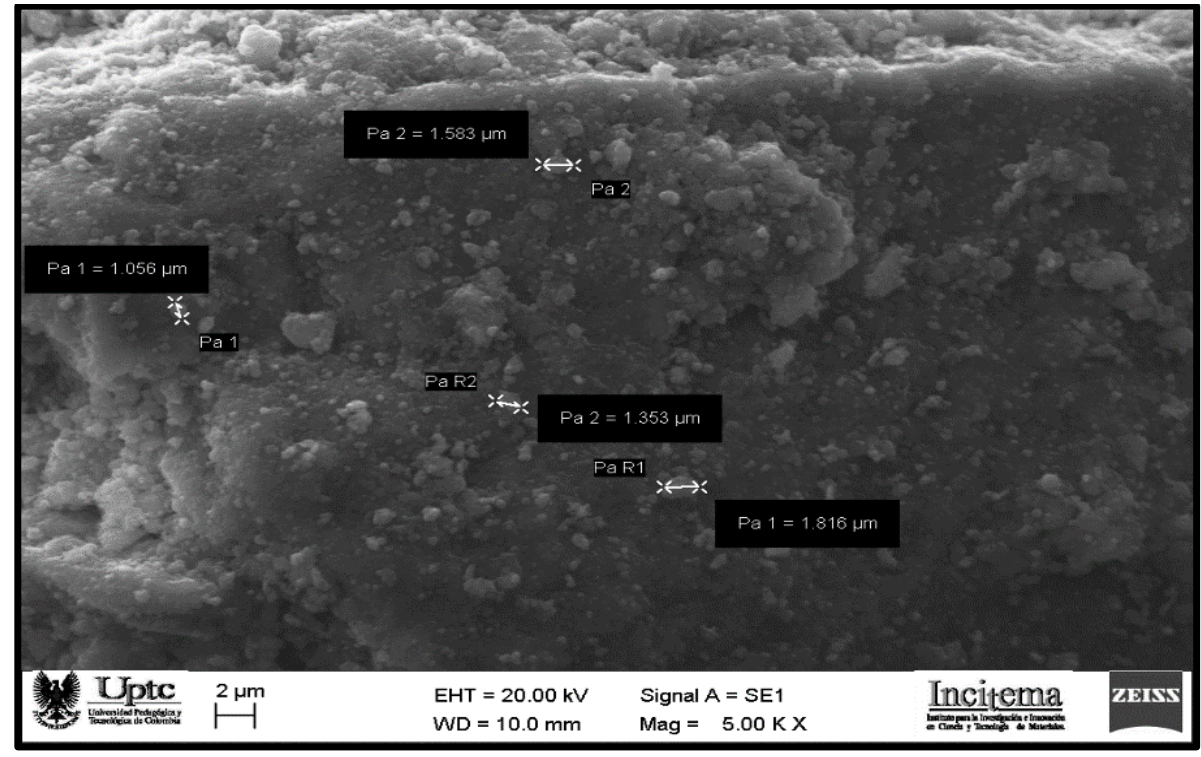

Adicionalmente, se reportó la composición porcentual elemental de RFIZA y RFPC, como muestras crudas, por energía dispersiva de rayos X (EDX), tal como se indica a continuación en las figuras 10 y 11:

\section{Figura 10.}

EDX: Energía dispersiva de rayos X para RFIZA.

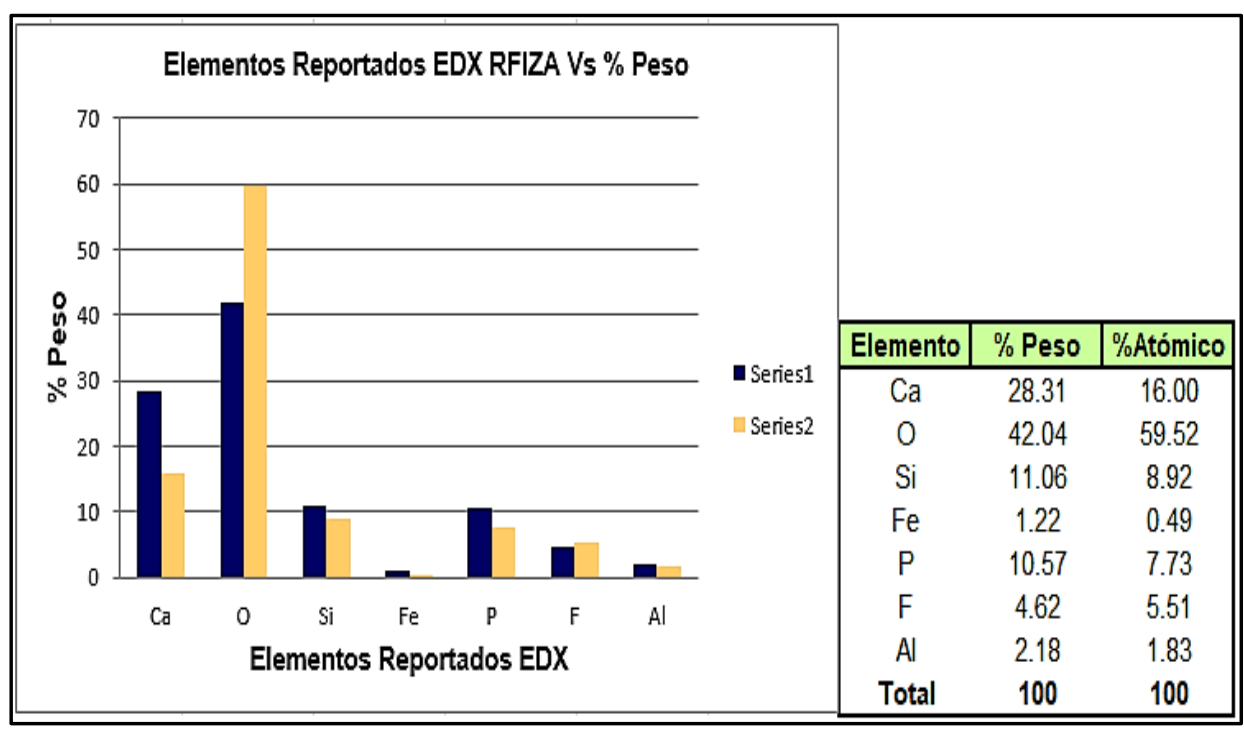


Figura 11.

EDX: Energía dispersiva de rayos X para RFPC.

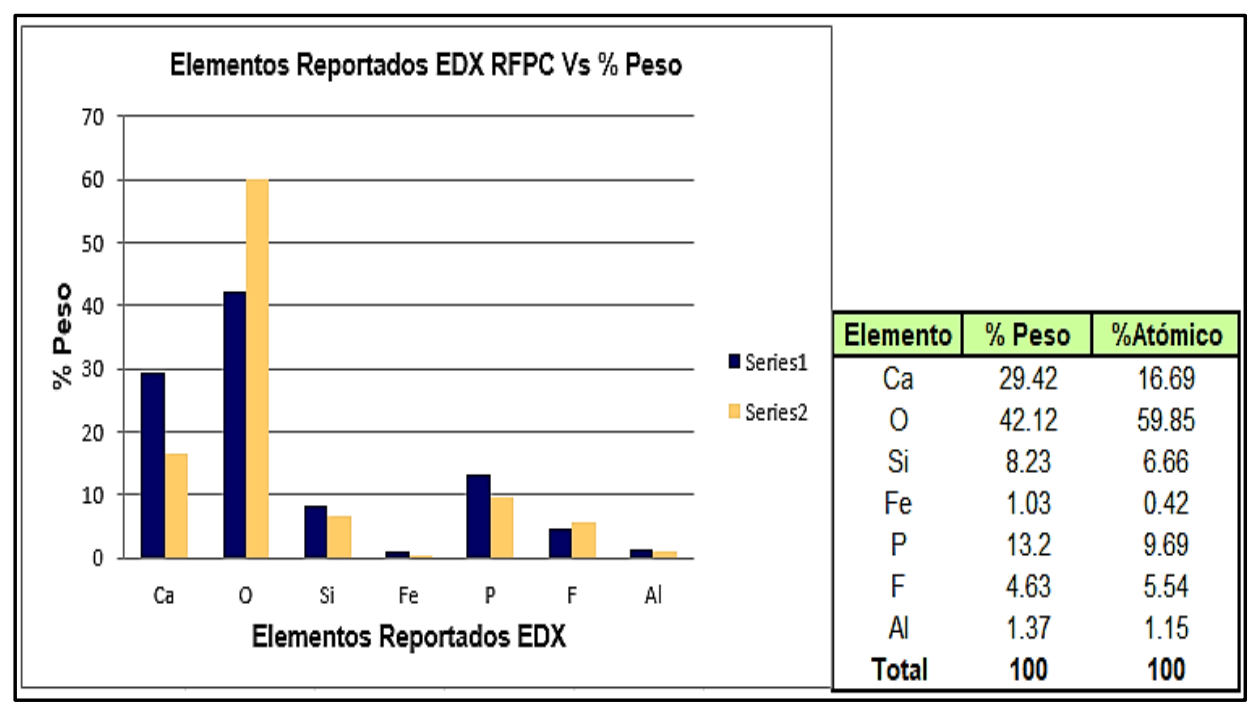

Los valores obtenidos mediante la técnica de EDX permiten determinar la relación $\mathrm{CaO} / \mathrm{P}_{2} \mathrm{O}_{5}$ para las muestras crudas reportadas en la tabla 9 , las cuales son mayores a 1.31; este incremento puede deberse a que cada sustitución de $\mathrm{PO}_{4}{ }^{=}$por $\mathrm{CO}_{3}{ }^{-}$o a la presencia de calcita o dolomita en la fosforita (Elgharbi et al., 2015).

\section{Tabla 9}

Cálculo de la relación $\mathrm{CaO} / \mathrm{P}_{2} \mathrm{O}_{5}$ por EDX

\begin{tabular}{lccc}
\hline Muestra & $\mathbf{C a O}$ & \% de $\mathbf{P}_{\mathbf{2}} \mathbf{O}_{\mathbf{5}}$ & $\mathbf{C a O} / \mathbf{P}_{\mathbf{2}} \mathbf{O}_{\mathbf{5}}$ \\
\hline RFIZA & 39,63 & 24,21 & 1,64 \\
RFPC & 41,19 & 30,23 & 1,36 \\
\hline
\end{tabular}

Para la determinación de humedad, materia volátil, cenizas y carbono fijo, se realizaron análisis termogravimétricos (TGA) en las muestras crudas RFIZA y RFPC, mediante un equipo marca LECO 701 y bajo la norma ASTM D7582. Los resultados se muestran en la tabla 10: 


\section{Tabla 10}

Análisis termogravimétricos (TGA) para las muestras crudas RFIZA y RFPC

\begin{tabular}{rcccc}
\hline Muestra & Humedad (\%) & Cenizas (\%) & Volátiles (\%) & C fijo (\%) \\
\hline RFIZA 1 & 1,14 & 97,18 & 1,43 & 1,39 \\
RFIZA 2 & 1,09 & 97,15 & 1,48 & 1,37 \\
RFPC 1 & 1,14 & 94,70 & 4,13 & 1,17 \\
RFPC 2 & 1,14 & 94,69 & 4,15 & 1,16 \\
\hline
\end{tabular}

Los análisis realizados mediante TGA indican que las muestras RFIZA y RFPC presentan un valor medio de $1,13 \%$ de humedad y las cenizas de RFIZA revelan mayores contenido de óxidos, los cuales se clasifican en óxidos básicos $\left(\mathrm{Fe}_{2} \mathrm{O}_{3}, \mathrm{CaO}, \mathrm{MgO}, \mathrm{Na}_{2} \mathrm{O}\right.$ y $\left.\mathrm{K}_{2} \mathrm{O}\right)$, óxidos ácidos $\left(\mathrm{SiO}_{2}, \mathrm{Al}_{2} \mathrm{O}_{3}\right.$ y $\left.\mathrm{TiO}_{2}\right)$ y otros óxidos $\left(\mathrm{SO}_{3}\right.$ y $\left.\mathrm{P}_{2} \mathrm{O}_{5}\right)$ (Peña Urueña, 2011). La RFPC presenta contenidos de cenizas menores, debido a un mayor porcentaje de compuestos volátiles.

Figura 12.

EDX: Energía dispersiva de rayos X para RFIZA.

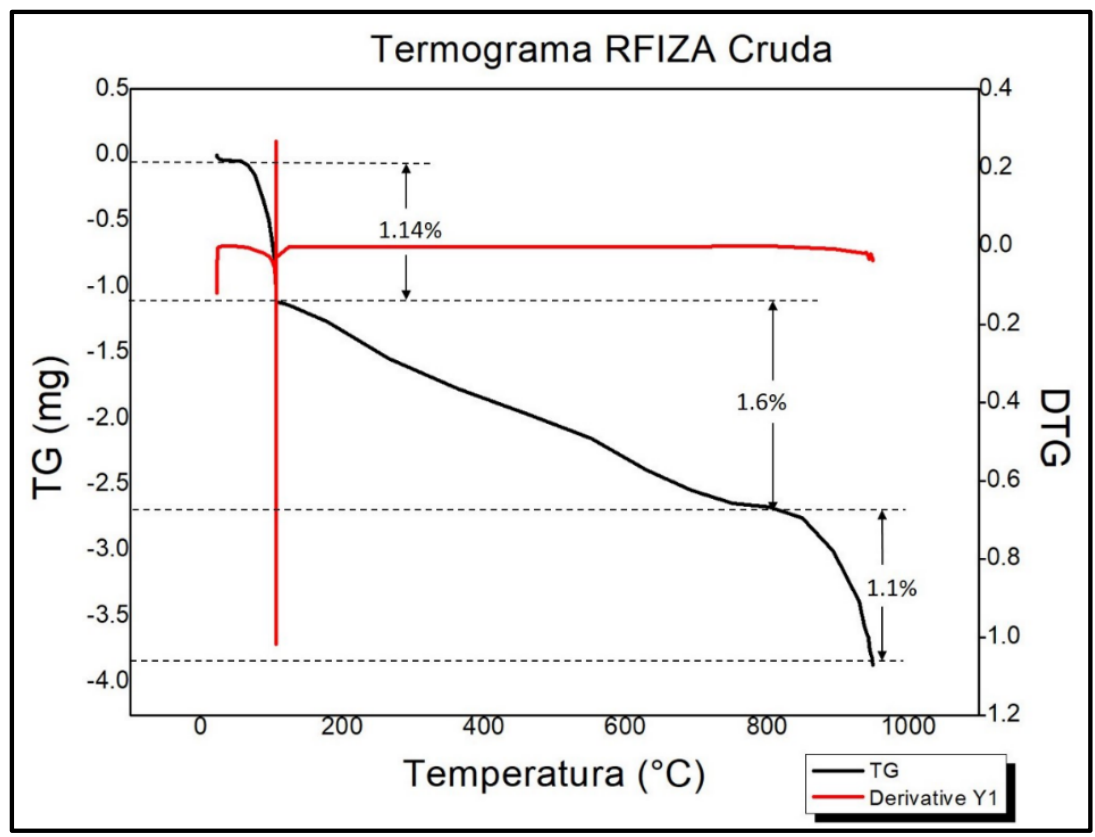

Nota. En la imagen se describe el comportamiento térmico de la muestra RFIZA cruda, para la determinación de humedad, cenizas y volátiles, aplicando la norma ASTM D7582. 


\section{Espinel Pérez}

La figura 12, muestra la curva TGA de RFIZA cruda, en la cual se presentan tres pérdidas de masa; la primera de ellas corresponde a un porcentaje de pérdida de masa de $1.14 \%$, entre 22 y $106.8^{\circ} \mathrm{C}$, asociado a la desorción de agua. La segunda es de $1.6 \%$ y la podemos encontrar entre $106.8^{\circ} \mathrm{C}$ y $803.5^{\circ} \mathrm{C}$, correspondiente a la zona transitoria de eliminación de materia orgánica y volátiles. La tercera es de $1.1 \%$, y se encuentra entre los $803.5^{\circ} \mathrm{C}$ y $990^{\circ} \mathrm{C}$; según Bachouâ et al. (2014), esta es atribuida a la descomposición de carbonatos presentes en los fosfatos. En el termograma diferencial (DTG), se corrobora la temperatura en la cual finaliza la primera pérdida de masa en la zona 1 , la cual corresponde a $106.8^{\circ} \mathrm{C}$.

Figura 13.

EDX: TGA RFPC Cruda: Termograma TG Vs Temperatura y Temperatura Vs DTG.

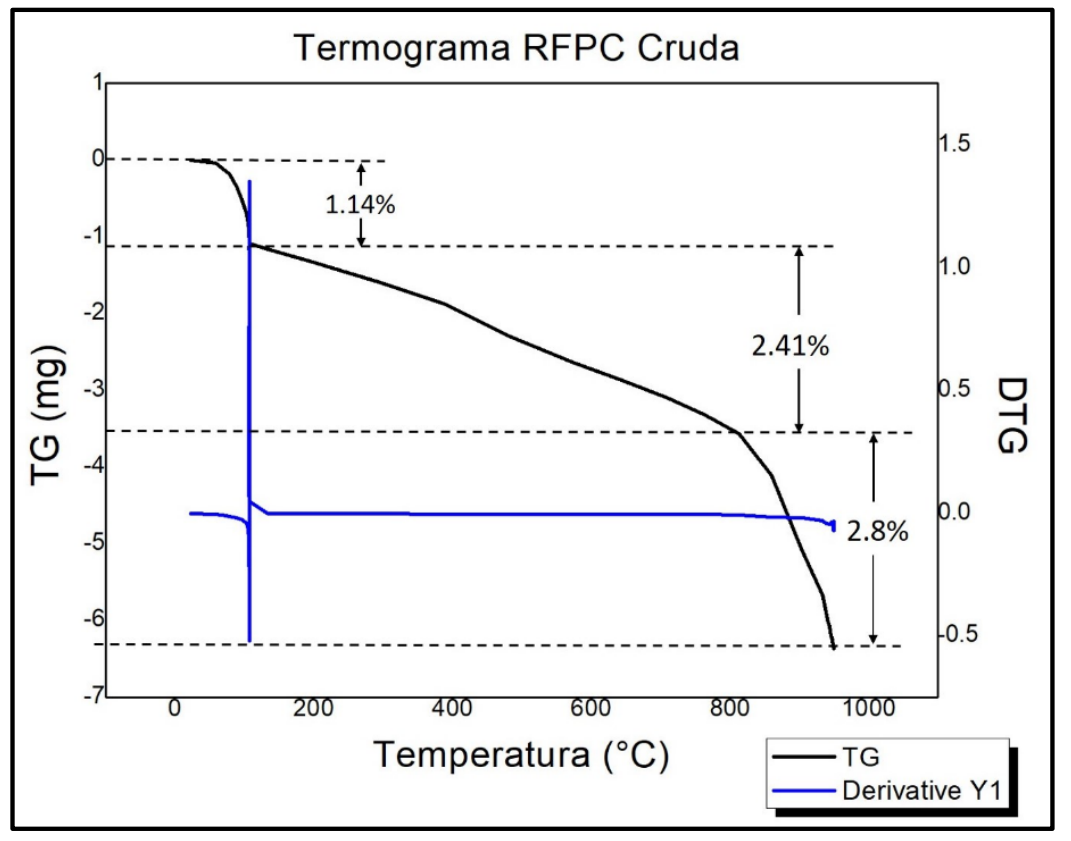

Nota. Las curvas de TGA y DTG realizadas para la RFPC Cruda.

Las curvas de TGA y DTG (figura 13) representan tres pérdidas de masa y corresponde a $1.14 \%$, entre los 22.57 y $107^{\circ} \mathrm{C}$, relacionada con la desorción de agua. La segunda es de $2.41 \%$, entre $107^{\circ} \mathrm{C}$ y $813.4^{\circ} \mathrm{C}$, presente en la zona transitoria de eliminación de materia orgánica y volátiles. La tercera es de $2.8 \%$ y se encuentra entre los $813.4^{\circ} \mathrm{C}$ y $949.3^{\circ} \mathrm{C}$, atribuida a la descomposición de carbonatos presentes en los fosfatos. En el termograma diferencial (DTG), se corrobora la temperatura en la cual finaliza la primera pérdida de masa en la zona 1 , la cual corresponde a $107^{\circ} \mathrm{C}$. 


\section{Conclusiones}

Las operaciones unitarias de reducción de tamaño y tamizado favorecen el enriquecimiento de $\mathrm{P}_{2} \mathrm{O}_{5}$ debido a sus fracciones finas.

Los termofosfatos obtenidos mediante hornos eléctricos, a partir de las muestras RFIZA y RFPC, incrementan su concentración de $\mathrm{P}_{2} \mathrm{O}_{5}$ cuando se someten a mayores tiempos de calcinación y a temperaturas superiores a los $1200^{\circ} \mathrm{C}$, obteniéndose mejores resultados (muestra E8), pero con costo energético superior.

Los análisis de DRX de los termofosfatos (TFHE-IZA y TFHE-PC), muestran que, la fase cristalina de fluoroapatita no desaparece a pesar de la calcinación, y esto se debe a una fuerte cohesión del flúor en este tipo de compuesto.

Las rocas fosfóricas de la región (Iza y Sogamoso) presentan una concentración entre el 23 y $28 \%$ de $\mathrm{P}_{2} \mathrm{O}_{5}$, pero aplicando procesos térmicos se pueden enriquecer para darle un mayor valor agregado.

Los incrementos de temperaturas aplicadas a las apatitas y del tiempo de calcinación, permiten una mayor conversión y remoción de agua, modificando la estructura cristalina de las mismas, facilitando la concentración de $\mathrm{P}_{2} \mathrm{O}_{5}$ y su solubilidad en citrato de amonio neutro.

La fluoroapatitas y cloroapatitas requieren mayores temperaturas para su descomposición, y esto se debe a la presencia de elementos electronegativos como el flúor y cloro, los cuales les dan mayor cohesión y estabilidad térmica a estos tipos de compuestos.

\section{Referencias}

Abouzeid, A. Z. (2008). Physical and Thermal Treatment of Phosphate ores - An Overview. International Journal of Mineral Processing, 85(4), 59-84. doi.org/10.1016/j.minpro.2007.09.001

Aissa , A., Abdeen , A., \& Abualreish , M. (2014). Qualitative and Quantitative Analysis of Phosphate Rock from Hazm Al-jalamid Area, Northern Saudi Arabia. International Journal of Basic and Applied Sciences, 3(3), 190-198. doi.org/10.14419/ijbas.v3i3.2755

Bachouâ, H., Othmani, M., Coppe, Y., Fatteh, N., Debbabi, M., \& Badraoui, B. (2014). Structural and Thermal Investigations of a Tunisian Natural Phosphate Rock. Journals materials and environmental science, 5(4), 1152-1159.

Bernal Gaona, S. (2013). Estudio de la calidad de recubrimientos de hidroxiapatita sobre acero inoxidable implantable aplicados mediante proyección térmica con plasma (Tesis Magister en Materiales y Procesos). Universidad Nacional de Colombia, Bogotá D.C. 
Espinel Pérez

Blazy, P., \& Bouhaouss, A. (2005). Removal of Organic Matter in Moroccan Youssoufia Phosphate by Flash Calcination. Minerals and Metallurgical Processing, 22, 107-115.

Bojinova, D. (2003). Thermal Treatment of Mixtures of Tunisian Phosphorite and Additives of Aluminum Silicate. Thermochimica Acta, 404(1-2), 155-162. doi.org/10.1016/S0040-6031(03)00145-X

Calle Castañeda, M. S. (2016). Evaluación de la acidulación de roca fosfórica empleando la bacteria acidófila Acidithiobacillus thiooxidans (Tesis Magister en Materiales y Procesos). Universidad Nacional de Colombia, Medellín.

El Ouardi, E. M. (2013). Effect of Temperature and Residence Time of Calcination Phosphate on the Chemical Reactivity: Application to the Case of Bouchane Phosphate (Morocco). International Journal of Innovation and Applied Studies, 4(2), 387-407.

Elgharbi, S., Horchani-Naifer, K., \& Férid, M. (2015). Investigation of the Structural and Mineralogical Changes of Tunisian Phosphorite during Calcinations. Journal of Thermal Analysis and Calorimetry, 119(1), 265.

El-Midany, A., El-Aleem, F., \& Al-Fariss, T. (2013). Why do Relatively Coarse Calcareous Phosphate Particles Perform Better in a Static-bed Calciner? Powder Technology, 237, 180-185.

Fahami, A., Bahman, N. T., \& Reza, E. K. (2013). Mechanosynthesis and Characterization of Chlorapatite Nanopowders. Materials Letters, 110, 117121. doi.org/10.1016/j.matlet.2013.08.012

Fernandez, S., y Noguera, R. (2003). Producción de fosfatos térmicos a partir de rocas fosfóricas nacionales. Agronomía Tropical, 53(1), 49-57.

Georecursos (2005). Análisis de la estructura productiva y mercados de la roca fosfórica. Georecursos.

International Fertilizer Association (2019). Short Term Fertilizer Outlook, IFA Strategic Forum. International Fertilizer Association.

Kijkowska, R., Lin, S., \& Legeros, R. Z. (2002). Physico-Chemical and Thermal Properties of Chlor-, Fluor- and Hydroxyapatites. Key Engineering Materials, 218-220, 31-34. doi.org/10.4028/www.scientific.net/KEM.218-220.31

Legeros, R. Z., Ito, A., Ishikawa, K., Sakae, T., \& Legeros, J. P. (2009). Fundamentals of Hydroxyapatite and Related Calcium Phosphates. En B. Basu, D. S. Katti \& A. Kumar (eds.), Advanced Biomaterials: Fundamentals, Processing, and Applications (pp. 19-52). John Wiley. doi.org/10.1002/9780470891315.ch2

Levingstone, T. J. (2008). Optimisation of Plasma Sprayed Hydroxyapatite Coatings (Tesis). Dublin City University, Ireland.

Norma Técnica Colombiana [NTC 234] (2001). Abonos o fertilizantes. Métodos de ensayo para la determinación cuantitativa de fósforo. Icontec. 
Peña Urueña, M. L. (2011). Caracterización de cenizas de algunos carbones colombianos in situ, por retrodispersión gamma-gamma. Universidad Nacional de Colombia.

Shariati, S., Ramadi, A., \& Salsani, A. (2015). Beneficiation of Low-Grade Phosphate Deposits by a Combination of Calcination and Shaking Tables: Southwest Iran. Minerals, 5, 367-379. doi:10.3390/min5030367

Straaten, P. V. (2002). Rocks for Crops, Agrominerals of Sub-Saharan Africa. Icraf.

Tõnsuaadu, K., Gross, K. A., Pluduma, L., \& Veiderma, M. (2012). A Review on the Thermal Stability of Calcium Apatites. Journal of Thermal Analysis and Calorimetry, 110, 647-659. doi.org/10.1007/s10973-011-1877-y

Van Kauwenbergh, S. J. (2010). World Phosphate Rock Reserves and Resources. Muscle Shoals, IFDC. Recuperado de:

https://pdf.usaid.gov/pdf_docs/Pnadw835.PDF 